\title{
Asociación de marcadores eritrocitarios (ABO, MNSs, Rh, Lewis, P1) con recurrencias y anomalías anatómicas y funcionales en niños con infección del tracto urinario
}

\author{
Patricio Herrera L. ${ }^{\text {; }}$ Rosanna Lagos Z. ${ }^{2}$; Alma Muñoz M. ${ }^{2}$; \\ Carmen Benavente R. ${ }^{2}$; Tamara Lamberg I. ${ }^{2}$; Inés Bravo R. ${ }^{1}$
Association of erytrhocyte markers (ABO, MNSs, Rh, Lewis, P1)
to recurrences, anatomical and functional abnormalities in children with urinary tract infections

\begin{abstract}
To contribute to the idenificotion of children with uringry rrect infection (UT) at increased risk of ecuirences or abrormol findings al imcge workup sludies (compications;, associations oekween these and red blood rells makers erithrocyle IABO. MNISs. Rh. Lewis, PI) distribution wəre studied ir 309 childrer with UTI. No associations were fourd between chy particular polymortism and complicalions, except for P1 blood ma'ser with E. cohr infection fOR = 3.07: ${ }^{\circ} 95 \%=1.13$ a 8.0; $\left.F<002\right]$ and the cbserke of other than $E$. coli agents in $B$ posilive polients $[C / 26$. Assence of ' $c$ r $E$. coli isolotions in phenotype $B$ soltents with UT', althougn nor stct sticolly significont, might sugest indepencert and odditive influence of $\mathrm{B}$ and $\mathrm{Pl}$ on determining the increased risk. as it has been shown for olher markers in U.'T. This associcton seems jiologically plausible, since both phenotypes have oeen separctely terated to LITl through uropithelial acherence phenolypical speriticing
\end{abstract}

Koy words: urinary lact infections, lower, Jpper, recurences, blood groups, iscanligens.l

Las infecciones del tracto urinario (infección urinaria) son importantes. por su frecuencia, complicaciones y significado pronóstico, aspectos de los cuales algunos han sido recientemente analizados en niños atendidos por el Servicio de Salud Metropolitano Norte de Santiago', ?. Dadas la escasez y coste de los recursos humanos y técnicos necesarios en el estudio y seguimiento de los niños afectados, se ha buscado información que permita ampliar la variedad de criterios usados habitualmente para identificar la población en mayor riesgo de complicaciones de esta enfermedad, y contribuir al empleo más eficiente de dichos recursos. Teniendo en cuen-

I. Departamento de Pediatría Norte y Centro de Epidemiología Clínica (INCLEN), Faculad de Medicuna, Universidad de Chile.

2. Hospital Roberto del Río, Servicio de Salud Metropolitano Norte.

Proyecto Fondecyt 19400-49. ta las posibles relaciones entre ciertos marcadores genéticos del huésped (polimorfismos de sistemas eritrocitarios) e infección urinaria, se exploró específicamente la posible asociación entre marcadores eritrocitarios de los polimorfismos $\mathrm{ABO}, \mathrm{Rh}, \mathrm{MNSs}$, Lewis y Pl y categorías clínicas de las infecciones urinarias (altas, bajas, recurrencias, asociación con alteraciones ultrasonograficas o radiologicas $)^{4-j}$, analizando -como hipótesis general-que en una población de niños con esta enfermedad, la distribución de los fenotipos determinados por esos polimor. fismos sería desigual entre individuos con y sin recurrencias o alteraciones anatomicas of funcionales de la vía urinaria.

\section{Pacientes y Métodos}

Se incluyó a 309 pacientes con infección urinaria entre los niños atendidos en una clínica especialmente organiza- 
da para tal propósito lus que han sido objeto ale anilisis clínicos y epidemiológicos anteriores' $y$ en los que se habia realizado identificación de los polimorfisnios erilrocitarios ABO, Rh. MNSs, Lewis, Pl y, adentás. cumplian los siguientes requisitos: haber sido seguidos por lo nenos un ines desde su primera atención por infección turnaria en la slínica al propósilo; contar con estudio de imánenes por ultrasonografía y uretrocisiografia miccional seriada: no tener controles previos ел el consultorio de tejrourología. El criterio de inclusión entre las infecciones del traclo uri. nario y sus categorías "alta" y "baja" han sido descritos en conunicaciones anteriores". Los criterios radiologicos usados fueron los habituales, reunidos para este análisis on las categorias "normal" $y$ "anormal". Là tipifuación de los antígenos erilrocitarios st hizo de acuerdo a técnicas esländar ${ }^{2}$

Para el análisis bioestadístico se usaron las proporciones como inedidas descriptivas: como medida de asociacion las ventajas o razones de disparidad (OR); como medida de dispersión los intervalos de contianza de $95 \%$ (IC $95 \%$ ) Comn pruebas de significación estajistica se usaron Ia de chi cuadrado o de probabilidad exacta de Fisher, segun fuese nectesurio. El analisis estratificido se hizo según el mélodo de Mante]-Haenzsele ${ }^{k}$. El nivel de signiticación fue estatieciclo en 10,05 .

\section{Resultados}

No se registraron asociaciones significativas directas entre alguno de los polimorfismos en particular y las categorías definidas por la locaJización (alta o baja) de la infección, probabilidad de recurrencias o alteraciones de los estudios aquí denominados radiológicos. Aunque sin llegar a la significación estadística, no se detectó la categoría etiológica "no coli". en los niños con tenotipo $\mathrm{B}$ del polimorfisino $\mathrm{ABO}$ (AB y BB) (0/26 casos), en cambio, se observó una frecuencia signiticativamente aumentada del lenotipo Pl, cuando el agente etiológico involucrado en la infección urinarja era $E$. coli $(\mathrm{OR}=3.07 ; \mathrm{IC} 95 \%=\mathrm{I}, 13$ a $8.6 ; \mathrm{p}<0,02)$ (tabla 1). En e] análisis estratificado, usando los polimorfismos previamente estudiados por scparado, no se constató interacción directa entre ellos con respecto a la probabilidad de complicaciones por las categorias de este fenotipo. Por ello, las relaciones entre Pl, etiología y fenotipo B se presentan en la tabla 2, en la que se analizan sólo los niños con etiología $E$. coli, grupo en el cual aparece mayor probabilidad de este agente asociada al fenotipo $\mathrm{Pl}$, en el grupo de niños no $B(O R=3,3$; JC $95 \%=1,2$ a $9,88: p<$ 0.02 ) (tabla 2). (B tendria efecto "protector" de recurrencias).

\section{Comentario}

Estc estudio de polimorfismos no permite identificar directamente subgrupos con riesgos diferenciales significativos de las características estudiadas de los pacientes con infeccion urinaria, pero ofrece algún apoyo a la hipótesis sobre la existencia de marcadores genéticos de posible utilidad clínica y epidemiológica. No debe extrañar la ausencia de asociaciones de polimorfismos que, por sí, determinen anomalías en los exámenes radiológicos, si se considera que el grupo estudiado en este análisis es seleccionado) pero no necesariamente representativo de las complicaciones de la infección urinaria. En cambio, la asociación de dos polimorfismos B y PI a la etiología $E$. coli es uno de los hechos esperados, aunque la primera to alcanza signiticación. La constatación de estas asociaciones ${ }^{46}$,

\section{Tabla 1}

Distribución del lénotipo eritrocitario

PI de los pacientes con infeccion del tracto urinario según la etiología de ésta

\begin{tabular}{|c|c|c|c|}
\hline & $\mathbf{P 1}(+)$ & $\begin{array}{l}\text { notipo } \\
\text { PI(-) }\end{array}$ & Total \\
\hline \multicolumn{4}{|l|}{ Etiología } \\
\hline $\begin{array}{l}\text { E. roli } \\
\text { No L Colj* }\end{array}$ & $\begin{array}{r}169 \\
7\end{array}$ & $\begin{array}{r}118 \\
1.5\end{array}$ & $\begin{array}{r}287 \\
22\end{array}$ \\
\hline Total & 176 & 133 & 309 \\
\hline
\end{tabular}

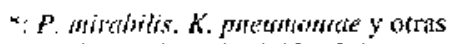
$O R=3.07$ IC $95 \%=1.13$ a 8.6$): p<0.02$

\section{Tabla 2}

Distribución del fenotipo eritrocitario P1 según la del fenotipo $B(A B O)$ en pacientes con infección del tracto urnario debida a $E$. Coli*

\begin{tabular}{lrrr}
\hline & PI $(+)$ & $\begin{array}{c}\text { Fenotipo P1 } \\
\text { P1 }- \text {-) }\end{array}$ & Total \\
\hline Fenotipo B & & & \\
B (-) & 154 & 100 & 254 \\
B $(+)$ & 7 & 15 & 22 \\
\hdashline- Total & 151 & 115 & 276 \\
\hline
\end{tabular}

$\mathrm{OR}=3,3(1 \mathrm{C} 95 \%=1,2$ a 9,9$) ; \mathrm{p}<0,0)$.

*: en la categoría "no E. roli" no hubu casos con fenotipo $B(+)$. 
sugirio la construccion del fenotipo combinando $P l$ y $B$. que no se tradujo en modificaciones de importancia de la relación entre PI y etiología de la infección urinaria. El tipo de interacción biológica sugerida por los resultados de cste análisis es interesante, pero no puede expresarsc en términos de bioestadística por ausencia de agentes etiológicos "no coli", en pa+ cientes con fenotipo $\mathrm{B}(\mathrm{ABO})$ ). Este último hallazgo es en sí llamativo, pues dicho fenotipo ha sido relacionado con la probabilidad de recurrir en mujeres adultas ${ }^{4}$, pero al carecer de significación estadística -probablementc por falta de poder del estudio- no permite mayores especulaciones. Pudiese ser que, en presencia de infección urinaria, la probabilidad de $E$. coli estaría facilitada por combinaciones fenotipicas B-P1, probablemente independientes $y$ eventualmente adilivas, como ha sido descrito para otros marcadores $^{6}$, pero no para estos lenotipos.

\section{Resumen}

Para contribuir a la identiticación de niños con infección del tracto urinario con mayor ricsgo de ubicación alta o baja, recurrencias y alteraciones radiológicas o ultrasonográficas (complicaciones) se estudiaron las asociaciones entre éstas y la distribución de marcadores eritrocitarios (ABO. MNSs, Rh, Lewis, P1), en 309 casos de infección urinaria. No se encontró asociación entre algún polimorfismo eritrocitario on particular con las mencionadas caregorías, pero sí entre el fenotipo P1 y la ctiología Escherichia coli $(\mathrm{OR}=3,07 ;$ IC $95 \%=1,13$ a 8,$6 ; \mathrm{p}<0,02)$ y la ausencia de etiología no $E$. coli con el fenotipo $\mathrm{B}+(0 / 26)$ sin llegar a niveles de significación. Estos hallazgos sugieren que, en niños con intección urinaria, estos fenotipos, por separado, probablemente tienen acciones independientes y aditivas.

(Palabras clave: infección del tracto urinario, alta, baja, recurencia, alteraciones estructurales, grupos sanguíneos, isoanticuerpos.)

\section{Referencias}

1. Lagts. $R$, Curter $J$, Herrera P: Liilidad de una tira reactiva y del aspecto macroscópico de la orina para descartar la sospecha de infeccion del tracto urinario en niños ambulatorios. Rey Chil Pediatt $1994 ; 65: 88-94$.

2. Lages $R_{1}$ Citrter J, Benusente $C$. et al: Epidemiologia de las infecciones del tracto urinario en niños y ricsgo de recursencias y alteraciones nefrourologicas. Rev Chil Pediut 1995: 66: 1-12.

3. Technical Manual of the American Associacion of Biood Banks, 9th DC Washington DC, 1985; 8: 118-120.

4. Crus-Coke $\mathcal{R}_{1}$ Paredes $L$, Monsenegro A: Blood groups and urinary micrnorganisins J Med Genet 1965: 2: J85188.

5. Lowberg H. Cedergren B. Leffler $H$. Nitsson B. Cartstroms A. Sitmborg-eden $C$ : Intluence of blood groups on the availability of receptors for attactinken of uropathogenic Exchesichis coli. Infect Immunl 1936; $51: 919-926$.

6. Rether $J$. Thondes $V$. Forland $M$ : Relationships between blood groups, bacterial pathogens and urinary urat infections. Am J Med Scj 1986; 292: 87.91.

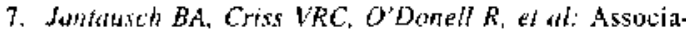
tion of Lewis blood group phenotypes with urinary tract infections in children. J Pediatr 1994; $124 ; 863-868$.

8. Schlessetmen $J I$ : Case control studies, Oxford University Press. NY 1982. 